\title{
Lévy Processes, Saltatory Foraging, and Superdiffusion
}

\author{
J. F. Burrow ${ }^{a}$, P. D. Baxter ${ }^{b}$ and J. W. Pitchford ${ }^{a, c, 1}$ \\ ${ }^{a}$ Department of Mathematics and York Centre for Complex Systems Analysis, \\ University of York, York, UK \\ ${ }^{b}$ Department of Statistics, University of Leeds, Leeds, UK \\ ${ }^{c}$ Department of Biology, University of York, York, UK
}

\begin{abstract}
It is well established that resource variability generated by spatial patchiness and turbulence is an important influence on the growth and recruitment of planktonic fish larvae. Empirical data show fractal-like prey distributions, and simulations indicate that scale-invariant foraging strategies may be optimal. Here we show how larval growth and recruitment in a turbulent environment can be formulated as a hitting time problem for a jump-diffusion process. We present two theoretical results. Firstly, if jumps are of a fixed size and occur as a Poisson process (embedded within a drift-diffusion), recruitment is effectively described by a diffusion process alone. Secondly, in the absence of diffusion, and for "patchy" jumps (of negative binomial size with Pareto inter-arrivals), the encounter process becomes superdiffusive. To synthesise these results we conduct a strategic simulation study where "patchy" jumps are embedded in a drift-diffusion process. We conclude that increasingly Lévy-like predator foraging strategies can have a significantly positive effect on recruitment at the population level.
\end{abstract}

Keywords: fish larvae, power law, Pareto distribution, hitting time, jump-diffusion, Lévy walk AMS subject classification: 92D25, 92D50, 60K40, 62M30

\section{Introduction}

Planktonic fish larvae may be broadly described as being small, stupid, and dead. These assertions can be made more concrete: larvae are small relative to the spatial scales of prey heterogeneity and to the turbulent fluid flow at these spatial scales [21]; they have only local knowledge of their

${ }^{1}$ Corresponding author. E-mail: jwp5@york.ac.uk 
immediate environment, limited by a visual perceptive distance of around one body length [22]; and they are subject to massive mortality, with a newly hatched individual's probability of survival to metamorphosis being $O(1 \%)$ or less [6] driven by typical mortality rates of $10 \%$ per day in the larval stage [9]. These factors are likely to strongly influence the extreme observed variability in the stock-recruitment relationships which underpin fisheries management.

Because the key natural phenomena are inherently stochastic, deterministic models can be argued to be inappropriate for quantifying recruitment (defined here as growth to some threshold size e.g. size at metamorphosis). Rather, stochastic models must be constructed to arrive at recruitment probabilities [21]. Recent recruitment models have treated this process as a hitting-time problem for stochastic differential equations $[17,23]$, showing that environmental stochasticity induced by turbulence and spatial heterogeneity can be beneficial to recruitment. The differences between the predictions from deterministic and stochastic models are particularly great when growth rates are small and mortality rates are large, which is precisely the environment inhabited by fish larvae [23].

The general approach of [17, 23], which assumes that individual-based variability can be captured at the population level by a diffusion equation, may not be universally appropriate. In particular, diffusion-based models cannot necessarily capture sudden jumps caused by rare chance encounters with particularly favourable patches of prey (there are parallels to the phenomenon of unpredictable shocks affecting the value of economic markets [1]). The Lévy-Khintchine formula provides a generic mathematical description for an infinitely divisible continuous time stochastic process as a combination of diffusion-with-drift interspersed with probabilistic jumps [1].

Important clues as to how the diffusion-based approach of $[17,23]$ could better account for planktonic heterogeneity have been provided by recent research on Lévy walks (LWs), with attempts to develop a single framework in which to study plankton patchiness [16] and non-Brownian motion of predators in heterogeneous stochastic environments [27]. A LW is a random walk with step lengths taken from an appropriate heavy tailed distribution [10], allowing very large steps ("jumps") to occur. The probability density function (pdf) for a LW typically takes the form of a power law; for example, $P\left(l_{j}\right) \sim l_{j}^{-\mu}$, with $1<\mu \leq 3$ where $l_{j}$ is the step length and $\mu$ is the Lévy exponent [27]. Brownian motion is recovered as a special case for $\mu>3$ [2, 24, 29]. Alternative parameterisations may be more amenable to mathematical progress (see Section 3).

Results from analytical and simulation models [3, 4, 28], supported by empirical data [27] suggest that a naive predator following a stochastic foraging strategy in a patchy prey environment can optimise its mean rate of prey encounters by following a LW. Furthermore, simulation results [27] suggest that a fitness benefit is conferred by following a Lévy foraging strategy the exponent of which matches that of the underlying prey distribution. These results are not supported by [5], who compares LWs with composite random walks (CWs) generated by a forager taking smaller steps when it perceives itself to be within a prey patch. Benhamou [5] shows firstly that a CW can outperform a LW in a patchy environment, and secondly that data sampled from CWs may resemble those from a LW, leading to possible problems in interpretation. Important questions also arise concerning pattern versus process in our understanding of animal movements: if a forager exploits a patchy prey environment by changing its movement strategy in response to its perceived prey field, then its movements will appear to be a stochastic LW foraging strategy when in fact 
they simply reflect the underlying prey distribution [5, 24].

This study seeks to provide a mathematical basis for the treatment of non-diffusive phenomena in descriptions of planktonic foraging. In Section 2 we address the question of whether a reformulation of the recruitment problem can account for locally rare but beneficial conditions using a jump-diffusion process. In Section 3 we are motivated by the saltatory (pause-travel) foraging behaviour of planktonic fish larvae (e.g., cod [25]). We use a deliberately simple analytical model to ask whether a saltatory strategy in a patchy environment is optimal, and whether there is a mathematical basis for the notion that predator and prey exponents should match. Our approach utilises analytically tractable Pareto distributions for step lengths and inter-arrival times [13]. Furthermore, the simplicity of the model means that pattern and process are transparently independent. The resulting individual-based model exhibits superdiffusivity; the variance of the process does not scale linearly with time.

The results in Section 3 support [5] and [21] in showing that Lévy foraging is not a generically optimal strategy. However, when synthesised within the Lévy process jump-diffusion framework of Section 2, results of strategic simulations lead us to argue in Section 4 that saltatory LW foraging may be a beneficial strategy when scaled up to the population level. We show that superdiffusivity can in principle increase recruitment probability due to the risk sensitivity associated with foraging in a high mortality environment. However, the ecological details peculiar to each foraging scenario (and therefore their simulation) are likely to be factors of major importance.

\section{Hitting times for jump diffusion processes and applications to recruitment}

Pitchford et al. [23] and Lv and Pitchford [17] show that including Gaussian white noise (representing individual and environmental variability) in the growth rate of planktonic fish larvae always increases the probability of maturation (defined here as growth to the recruitment threshold size). Pitchford et al. [23] describe the gain in mass $M(t)$ of an individual larva at time $t$ as

$$
M(t)=r t+\sigma B(t), \quad M(0)=0,
$$

so that each larva grows as a drift-diffusion process with mean rate $r$ and with variance $\sigma^{2}$.

Because it forms a basis for our subsequent analysis, the model of (2.1) deserves careful consideration, especially regarding linearity in time $t$ and the possibility of negative growth. Modifying the drift term $r$ to account for concave (Von Bertalanffy growth) or convex (geometric) growth, and allowing different scaling of the noise term $\sigma$, does not qualitatively affect the results for recruitment probabilities [17]. Equation 2.1 admits the possibility of $M(t)$ becoming negative. However, if $M(t)$ is interpreted as a measure of gain in mass from an initial non-zero state $M_{0}$, then $M(t)<0$ does not necessarily imply that the overall mass is negative. The probability of $M(t)<-M_{0}$ (implying negative mass) for some $t<\infty$ is $\exp \left(\frac{-2 r M_{0}}{\sigma^{2}}\right)$ (assuming no absorbing barrier at $\left.M_{\text {mat }}\right)$. The possibility that $M(t)<0$ is addressed fully in Appendix A. 
In light of the Lévy-Khintchine formula [1] and the patchy nature of plankton distributions [16], we quantify the effects of adding non-Gaussian (Lévy) noise which could represent prey patchiness, turbulence, or any other processes causing temporal and spatial heterogeneity. Consider the simplest case, where the Lévy measure $v$ takes the form $v=\lambda \delta_{h}$, with $\lambda>0$ and $\delta_{h}$ a Dirac mass centered at $h \in \mathbb{R} \backslash\{0\}$ [1]. This gives a new growth equation,

$$
M(t)=r^{\prime} t+\sigma B(t)+N(t), \quad M(0)=0,
$$

where $r^{\prime}=r-\int_{(0,1]} x v(d x)$ is the effective growth rate, $\sigma^{2}$ is the variance of the Brownian motion $B(t)$, and $N(t)$ is a Poisson random variable of intensity $\lambda$ taking values in $\{n h, n \in \mathbb{N}\}$, with

$$
P(N(t)=n h)=e^{-\lambda t} \frac{(\lambda t)^{n}}{n !}
$$

Equation (2.2) describes a fish larva growing with drift $r^{\prime}$ and variance $\sigma^{2}$, between jump discontinuities of size $h$, caused by encountering rare but favourable patches of prey which occur at random times $\left(T_{n}, n \in \mathbb{N}\right), T_{n} \sim \operatorname{Exp}(\lambda)$.

As in [23] we are interested in the probability of a fish larva reaching maturation. We first determine the distribution of the time to maturation, $t_{m a t}$, where

$$
t_{m a t}=\inf \left\{t>0: M(t)=M_{m a t}\right\} .
$$

$M_{\text {mat }}$ is defined to be the fixed recruitment mass and $t_{m a t}$ is known as a hitting time [7]. Using Theorem 1.1 of [19], the hitting time density for equation (2.2) can be shown to be

$$
f_{t_{m a t}}(t)=\frac{M_{m a t}}{t} \sum_{n=0}^{\infty} \frac{e^{-\lambda t}}{\sqrt{2 \pi \sigma^{2} t}} \frac{(\lambda t)^{n}}{n !} \exp \left[\frac{-\left(M_{m a t}-n|h|-r t\right)^{2}}{2 \sigma^{2} t}\right] .
$$

Although alternative possibilities exist $([9,20])$, a size-independent mortality process is appropriate and parsimonious (see [23]) i.e. mortality occurs as a Poisson process with rate $\mu$, so that an individual fish larva has a probability $\exp \left(-\mu t_{m a t}\right)$ of surviving to $M_{\text {mat }}$. Hence the probability of an individual reaching maturity is

$$
\begin{aligned}
P_{\text {mat }}(r, \sigma, \lambda, h) & =\int_{0}^{\infty} f_{t_{\text {mat }}}(t) \exp (-\mu t) d t \\
& =\frac{M_{\text {mat }}}{\sqrt{2 \pi \sigma^{2}}} \int_{0}^{\infty} \frac{e^{-(\mu+\lambda) t}}{\sqrt{t^{3}}} \sum_{n=0}^{\infty} \frac{(\lambda t)^{n}}{n !} \exp \left[\frac{-\left(M_{\text {mat }}-n|h|-r t\right)^{2}}{2 \sigma^{2} t}\right] \mathrm{dt} .
\end{aligned}
$$

To investigate the effects of the additional Lévy noise in the linear growth model, the recruitment probability is plotted against mortality rate $\mu$ for a pure drift-diffusion growth process, a mixed drift-diffusion-jump growth process, and a pure Poisson jump growth process (Figure 1). The equation for the recruitment probability for the mixed drift-diffusion-jump growth process is 
given by equation (2.3), and the equation for the pure drift-diffusion process is given by Equation (8) in [23]. The probability of maturation for a pure Poisson jump growth process, with intensity $\lambda$ and jump size $h$, is

$$
P_{m a t}(\lambda, h)=\left(\frac{\lambda}{\lambda+\mu}\right)^{\frac{M_{m a t}}{h}}
$$

The analytical results in Figure 1 were confirmed using explicit individual-based simulations of 100,000 individuals generated using an Euler-Maruyama scheme between exponentially distributed fixed size jumps.

a)

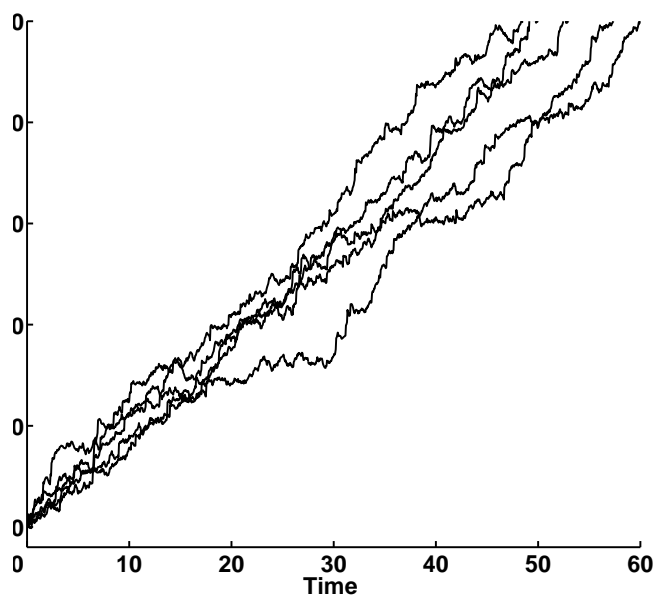

b)

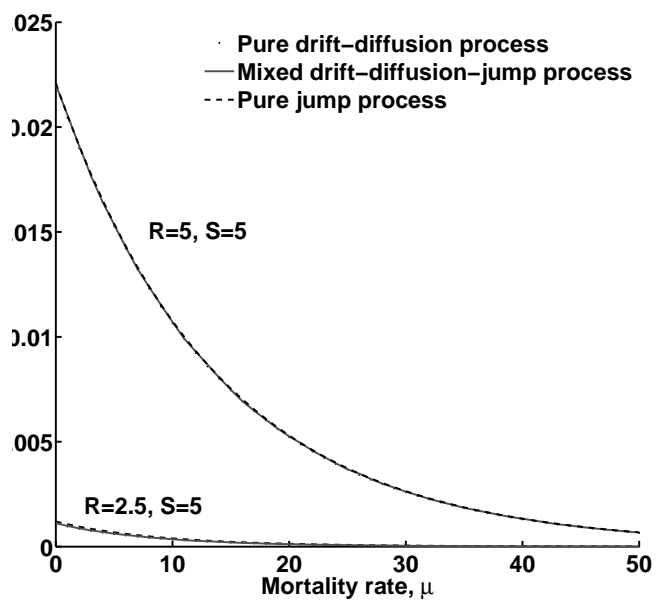

Figure 1: a) Example growth trajectories for individuals growing with a drift-diffusion process between exponentially distributed fixed size jumps. Parameters used here were $r=2.5, \sigma^{2}=12.5$, $h=5, \lambda=0.5$. b) The probability of reaching maturity against mortality rate $\mu$, for a pure driftdiffusion growth process, a mixed drift-diffusion-jump growth process, and a pure Poisson jump growth process. The mean at time $t$ for all processes was fixed to be $R t$, and the variance $S^{2} t$. For the drift-diffusion process the parameters used were $(R=5) r=5, \sigma=5$; and $(R=2.5) r=2.5$ and $\sigma=5$. For the mixed drift-diffusion-jump process the parameters used were, $(R=5) r=2.5, \sigma=\sqrt{12.5}, h=5$, $\lambda=0.5$; and $(R=2.5) r=1.25, \sigma=\sqrt{12.5}, h=10, \lambda=0.125$. For the pure jump process the parameters used were $(R=5) h=5, \lambda=1$; and $(R=2.5) h=10, \lambda=0.25$.

The results in Figure 1 indicate that, at the scale of larval fish growth, the addition of Lévy noise (in the form of constant size jumps) is unlikely to affect the probability of maturation when the mean and variance of the overall growth process are held fixed. In other words, at the ecologically relevant scale, the input from the jump process becomes essentially diffusive and standard stochastic differential equation (SDE) techniques can be applied. The following section shows 
that this is a consequence of the choice of jump process, and that more realistic foraging models can result in non-diffusive processes at the population level, with concomitant consequences for recruitment.

\section{An individual-based model for the encounter process of a saltatory forager}

The analytical and numerical results in Section 2 indicate that simply describing individual-based growth and recruitment as a hitting time problem for a jump-diffusion process does not necessarily scale up to have an impact at the ecological or management levels. However, the assumption that jumps occur with a fixed magnitude is unrealistic and may be unnecessarily restrictive, because the resulting model resembles a diffusive process over ecologically relevant time scales. The individual-based model formulated below provides theoretical evidence that diffusive models may be inappropriate; for a saltatory forager following a LW in a patchy environment, it is demonstrated that the growth process is superdiffusive.

Consider a naive predator (e.g., planktonic fish larva) foraging in a patchy environment where food items (e.g., copepods) are distributed patchily in space according to a Pareto distribution with parameter $r_{2}$ [14]. The predator performs a saltatory foraging strategy, moving between search locations at a fixed constant speed. These movements have lengths governed by a Pareto distribution with parameter $r_{1}$, i.e., the step lengths are such that the predator follows a LW for $r_{1} \leq 2$. We assume that the predator consumes all items of food it finds within each search location before moving on to its next foraging location, and that this consumption is instantaneous (i.e. there is a negligible handling time). These assumptions are useful for analytical tractability, and could be relaxed in numerical simulations.

Although we assume a three dimensional isotropic prey distribution, the foraging process can be regarded as taking place in one spatial dimension (c.f. [24]). This is not a restrictive assumption because the movement process is one dimensional from the forager's point of view and Lévy exponents are conserved when dimensionality is reduced via projection [27] (Suppl. Mat.). In order to understand how the spatial distribution of predator foraging locations and prey items may be interrelated, we parameterise the probability distributions such that the expected number of foraging locations and the mean-field prey density remain fixed, and only the degree of heterogeneity (of forager movements, prey distribution, or both) varies. This focuses attention on the foraging strategy employed, rather than any mean-field properties. Note that this does not imply a fixed prey field. Conditional on a fixed mean-field density, the number of prey items at each location is independent of all other locations.

We seek to understand the distribution of $X(t)$, the total number of prey items encountered by a stochastic forager in fixed time $t$. Let $N(t)$ be the number of foraging locations visited in time $t$, and let $\delta$ describe the size of each foraging location (typically the perceptive radius of the forager [21]). Because the forager travels with constant speed, the time $\tau$ between foraging intervals follows a Pareto distribution with parameters $r_{1}$ and $\alpha_{1}$. Hence the probability density of 
$\tau$ is given by

$$
\mathrm{f}(\tau)=\frac{r_{1} \alpha_{1}^{r_{1}}}{\left(\alpha_{1}+\tau\right)^{r_{1}+1}}, \quad(\tau>0)
$$

It follows that [13]

$$
N(t) \sim \text { negative binomial }\left(r=r_{1}, p=\frac{\alpha_{1}}{\alpha_{1}+t}\right),
$$

with probability mass function

$$
\mathbf{f}(n)=\left(\begin{array}{c}
r+n-1 \\
n
\end{array}\right) p^{r}(1-p)^{n}, \quad(n=0,1, \ldots) .
$$

For foraging location $i$, let the number of encounters with prey be $Y_{i}$, i.e, $Y_{i}$ is the number of prey items contained within a sphere of radius $\delta$. Because the prey are distributed according to a Pareto distribution with parameters $r_{2}$ and $\alpha_{2}$, the distance $\eta$ between prey items has density function

$$
\mathbf{f}(\eta)=\frac{r_{2} \alpha_{2}^{r_{2}}}{\left(\alpha_{2}+\eta\right)^{r_{2}+1}}, \quad(\eta>0)
$$

and it follows that

$$
Y_{i} \sim \text { negative binomial }\left(r=r_{2}, p=\frac{\alpha_{2}}{\alpha_{2}+\delta}\right) .
$$

Hence the probability mass function of $Y_{i}$ is

$$
\mathbf{f}(y)=\left(\begin{array}{c}
r+y-1 \\
y
\end{array}\right) p^{r}(1-p)^{y}, \quad(y=0,1, \ldots) .
$$

We make the natural assumption that the $Y_{i}$ are independent and identically distributed. There is an implicit but practically reasonable assumption here that foraging locations do not overlap [18]. The fact that the perceptive field of a larva is better described as a narrow "wedge" further strengthens this argument [12]. The probability generating functions of $N$ and $Y_{i}$ can then be derived as

$$
\begin{aligned}
& G_{N}(s)=\left(\frac{\alpha_{1}}{\alpha_{1}+[1-s] t}\right)^{r_{1}}, \\
& G_{Y}(s)=\left(\frac{\alpha_{2}}{\alpha_{2}+[1-s] \delta}\right)^{r_{2}} .
\end{aligned}
$$

It follows that $\mathrm{E}(N)=r_{1} t / \alpha_{1}$ and $\mathrm{E}\left(Y_{i}\right)=r_{2} \delta / \alpha_{2}$.

Because the total number of prey encountered in time $t$ is simply

$$
X=Y_{1}+Y_{2}+\cdots+Y_{N}
$$

it follows that the generating function of $X$ is

$$
G_{X}(s)=G_{N}\left\{G_{Y}(s)\right\}=\alpha_{1}^{r_{1}}\left[t-t\left(\frac{\alpha_{2}}{\alpha_{2}-[1-s] \delta}\right)^{r_{2}}+\alpha_{1}\right]^{-r_{1}} .
$$


Let $\lambda_{1}=r_{1} / \alpha_{1}$ and $\lambda_{2}=r_{2} / \alpha_{2}$. By parameterising in terms of $r_{1}, r_{2}, \lambda_{1}, \lambda_{2}$, we are able to vary the parameters $r_{1}$ and $r_{2}$ whilst $\mathrm{E}(N)=\lambda_{1} t$ and $\mathrm{E}\left(Y_{i}\right)=\lambda_{2} \delta$ (mean-field properties) remain fixed. It can then readily be shown that

$$
\begin{aligned}
\mathrm{E}(X) & =\lambda_{1} \lambda_{2} \delta t \\
\operatorname{Var}(X) & =\lambda_{1} \lambda_{2}^{2} \delta^{2} t^{2}\left(\frac{1}{r_{1}}+\frac{1}{r_{2} t}+\frac{1}{t}+\frac{1}{\lambda_{2} \delta t}\right) .
\end{aligned}
$$

The implications of these results are discussed in more detail in Section 4, but the most basic message here is that the expected encounter rate depends only on the mean-field properties of the predator movement and prey distribution, not on the details of their distribution (i.e., $E(X)$ is independent of $r_{1}$ and $r_{2}$ ). This precisely mirrors the results of [21] and [22] for cruise predators: for both cruise and saltatory foragers, heterogeneity in the predator's movement strategy, or in the prey distribution, or both, do not affect mean prey encounter rates. Hence a constant speed predator receives no average benefit by altering its foraging trajectory (although changes in speed can of course influence encounter rates and movement costs [22]).

The variance in the encounter rate is superdiffusive $\left(\operatorname{Var}(X) \propto t^{\rho}, 1<\rho<2\right)$. Because the model in this section relates to encounter rate rather than time to maturity, it is not straightforward to incorporate mortality and formulate a model for recruitment probability as in Section 2. In Section 4.1, we incorporate the features of the "patchy" jump process that leads to superdiffusion into an idealised simulation of a jump-diffusion process. The consequences for recruitment are then discussed.

\section{Discussion}

The analytical results in Sections 2 and 3 raise important issues regarding both the inclusion of stochasticity in mathematical models, and the ecological and evolutionary processes underpinning our ideas of planktonic foraging.

\subsection{Superdiffusive models of recruitment}

Motivated by Sections 2 and 3, Figure 2 shows the results of simulating a jump-diffusion process with Pareto interarrival times between jumps (simulating a saltatory predator) and with negative binomially distributed jump sizes (simulating a patchy prey distribution). Parameter values are again chosen based on [23] so far as possible, and mortality rate $\mu$ is fixed at 0.1. The mean growth rate of the overall stochastic process is $R=r+\lambda_{1} \lambda_{2}$. Consistent with Section $2, R=5$, $r=2.5$ and $M_{\text {mat }}=200$. Results are shown for a range of $r_{1}$ and $r_{2}$, to capture the dependence of recruitment probabilities on both the foraging strategy of the predator and the patchiness of the prey distribution. A minimum exponent value $r_{i}=2$ is imposed because smaller values correspond to infinite variance and are therefore difficult to justify with empirical data. The results 
are calculated from simulations of 10,000 individuals using an Euler-Maruyama scheme between Pareto distributed negative binomial jumps.

Figure 2a) depicts five example individual growth trajectories for $r_{1}=2, r_{2}=2$, illustrating the non-diffusive nature of the underlying process. The consequences at the population level are shown in Figure 2b); recruitment probability is seen to increase with decreasing exponent $r_{1}$. The role of the prey distribution, captured by prey exponent $r_{2}$, appears to be less important (given that the mean-field prey concentration is constant across all simulations). However, whilst illustrating the general principle, the simulations in Figure 2 ought to be regarded as strategic. The Pareto formulation in Section 3 allows many small jumps rather than enforcing a minimum jump size. This may be inappropriate for some predators (although the factors of turbulence and wedge-shaped perceptive fields may ameliorate this criticism; the assumption that foraging locations are disjoint is likely to be reasonable [18]). Practical applications of this modelling framework would require context-specific consideration of underlying processes such as turbulence, swimming speeds and handling times and are beyond the scope of this study.

a)

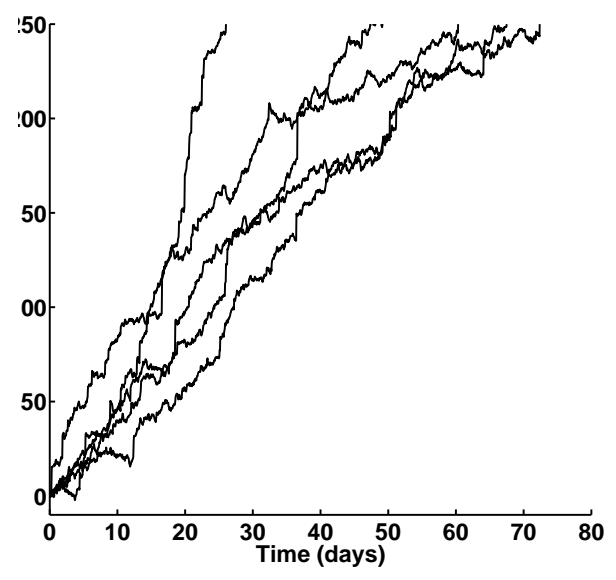

Figure 2: a) Example superdiffusive growth trajectories for individuals growing with a driftdiffusion process between negative binomially distributed jumps at Pareto distributed inter-arrival times. Parameters used here were $r=2.5, \sigma^{2}=12.5, r_{1}=2, \alpha_{1}=2, r_{2}=2, p=2 / 7$. b) The probability of reaching maturity against predator Pareto exponent, $r_{1}$, for a range of values of prey patchiness, $r_{2}$. Mass at maturation was fixed at 200, and mortality rate at 0.1 . The mean at time $t$ for all processes was fixed to be $R t=\left(r+\lambda_{1} \lambda_{2}\right) t=5 t$ (see Sections 2 and 3 for definitions of variables).

\subsection{Foraging behaviour}

Important messages arise from the encounter process modelled in Section 3. By employing increasingly non-diffusive Lévy-type movements (i.e., decreasing $r_{1}$ ), the predator can increase the 
variability in its encounter rate. Decreasing $r_{2}$ increases the variability in encounter rates at the individual level. In other words, although mean encounter rates are not affected, prey patchiness and Lévy foraging increase the variability in the gain an individual forager experiences. This echoes the results of [21], and it is possible to combine the results presented here with those of [5] and [21] to make some definite statements regarding stochastic foraging. Where simulations show a fitness benefit (an increase in mean encounter rate) in Lévy foraging over RW foraging, then the benefit does not arise solely as a consequence of predator movement and prey patchiness. Rather, where any benefit exists, it must arise in conjunction with other processes within the simulations. Details such as predators' behavioural responses to prey, how prey regeneration is handled, and how prey patchiness is statistically maintained after a predation event, are likely candidates. Such processes warrant greater attention to biological and ecological detail in order to build a more comprehensive picture of "optimal" foraging.

Predator movement and prey heterogeneity alone are not sufficient to favour Lévy foraging. Benhamou [5] suggests that, where LW are observed in data (and provided these have not been misidentified [10, 26, 27]), this is likely to be a confusion of pattern and process or a superposition of different movement strategies operating at different spatial scales. There is, however, another possibility which may be of particular importance for marine plankton (zooplankton or larval and juvenile fish) subject to high levels of mortality and turbulence. Pitchford et al. [23] show that stochasticity, manifested by a large variance in individual prey encounter rate, is beneficial to the growth and recruitment of organisms which grow slowly and whose survival to the next life history stage is unlikely (see [17] for nonlinear generalisations of this simple model). Such foragers, whose knowledge of their turbulent environment is necessarily local in both space and time, have a strong evolutionary pressure to increase their encounter variance (i.e., to follow a classic "risk sensitive" foraging strategy). It is not the object of this paper to quantify the evolutionary consequences of risk sensitivity, but we note that the model of evolutionary stable strategies under uncertain tradeoffs in [8] provides an appropriate framework. The deterministic energy costs of faster swimming must be balanced against the stochastic benefits of increased mean and variance in encounter rate, which can be quantified using the mathematics in Section 3 (saltatory predators) or [21] (cruise predators).

The individual-based foraging model presented here is highly idealised, but this is necessary to disentangle the "pattern versus process" arguments described in [5]. In particular, the model implies that the forager will not interact with any prey it meets whilst moving between foraging locations. Adapting the model to account for such possibilities inevitably leads one to consider more cruise-like foraging strategies where the theories of $[5,21,29]$ are more appropriate. We note that, compared to a saltatory forager, cruise predation is unlikely to be an effective way to leave regions of low prey density, i.e., to escape low quality habitats. The ability to leave regions of low yield in favour of a small chance of finding a higher yield location is likely to be of particular importance to risk sensitive foragers. Therefore, although some of the complex behaviours observed in saltatory foraging are missing [25], our model can be claimed to capture and quantify the fundamental processes. 


\subsection{Summary}

This study attempts to synthesise observational evidence of the non-diffusive distribution of planktonic predators and their prey in the natural environment with existing stochastic models, thereby characterising important ecological processes at the population level. We show that, when generalising from SDE to jump-diffusion process, the individual-level processes which generate the jump distribution can give rise to superdiffusivity.

The model of saltatory foraging in Section 3 agrees with previous models of cruise predators in questioning whether there is a generic advantage, in terms of mean encounter rate, for a naive predator to move according to a LW. Furthermore, we find no theoretical support for matching between exponents governing predator and prey distributions in maximising mean prey encounter rates. However, when interpreted in the context of a risk sensitive foraging strategy in a patchy environment, Figure 2 shows that saltatory foragers may be at a significant advantage. Accurately quantifying this advantage requires more careful consideration of the ecological details (minimum jump size, prey regeneration, predator perceptive field and behaviour, handling time etc.). These form the subject of ongoing work.

\section{Acknowledgements}

The authors thank Simon Benhamou, Edward Codling, Joe Horwood, Alex James, and Mike Plank for valuable input. The authors also thank three anonymous reviewers whose comments and criticisms have greatly improved this paper. JFB is supported by a PhD studentship financed by EPSRC and CEFAS.

\section{Appendix A: A double barrier hitting time problem}

To address the possibility of negative mass in the model of [23], we can redefine the recruitment problem as a double barrier hitting time problem. The gain in mass equation

$$
M(t)=r t+\sigma B(t), \quad M(0)=0,
$$

still holds, however in addition to the absorbing barrier at $M_{m a t}$, we now place another absorbing barrier at $-M_{0}$, where $M_{0}$ is defined to be the initial mass of a single planktonic fish larva. We wish to find the hitting time distribution for $M_{m a t}$ given that the growth trajectory $M(t)$ does not hit the barrier at $-M_{0}$ earlier. Using the methods of [15], we can derive this distribution:

$$
f_{\hat{t}_{\text {mat }}}(t)=\exp \left\{\frac{M_{\text {mat }} r}{\sigma^{2}}-\frac{1}{2}\left(\frac{r}{\sigma}\right)^{2} t\right\} \sum_{n=-\infty}^{\infty} g\left(t ; b_{n}\right),
$$

where

$$
g(t ; x)=\frac{x}{\sqrt{2 \pi t^{3}}} e^{-\frac{x^{2}}{2 t}}, \quad b_{n}=\frac{1}{\sigma}\left(2 n\left[M_{m a t}+M_{0}\right]+M_{m a t}\right),
$$


and

$$
\hat{t}_{\text {mat }}=\inf \left(t>0: M(t)=M_{\text {mat }} \mid M(s)>-M_{0}, 0 \leq s \leq t\right)
$$

is the redefined hitting time.

a)
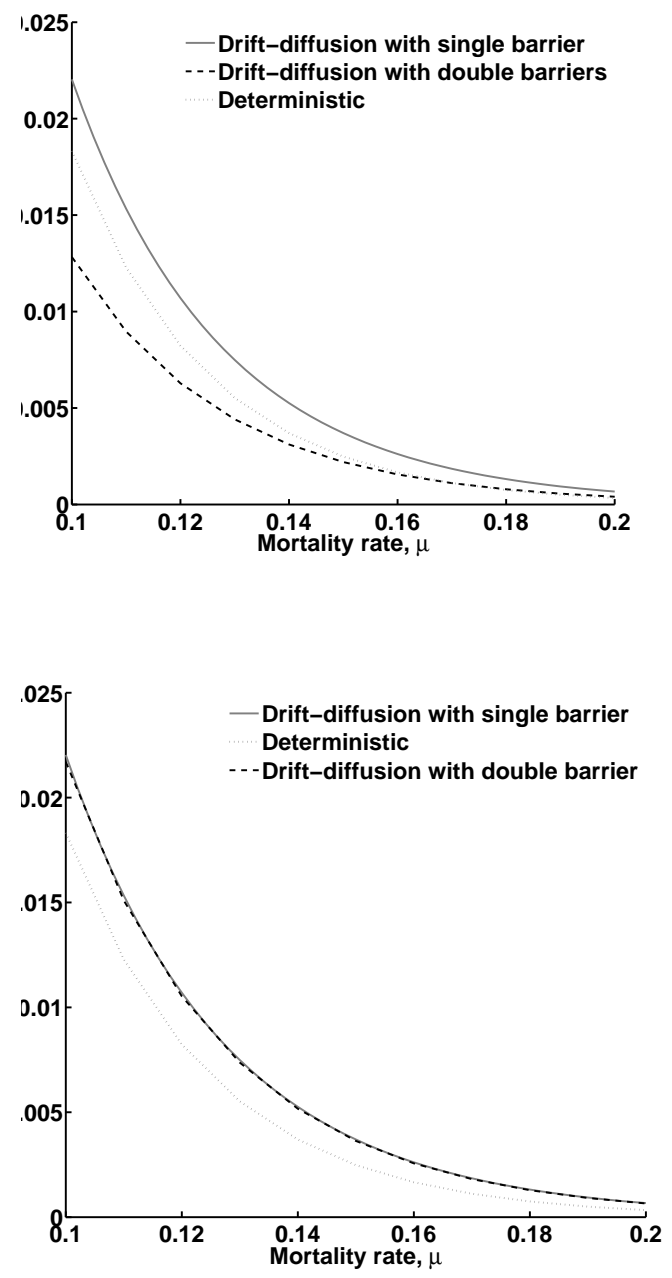

b)

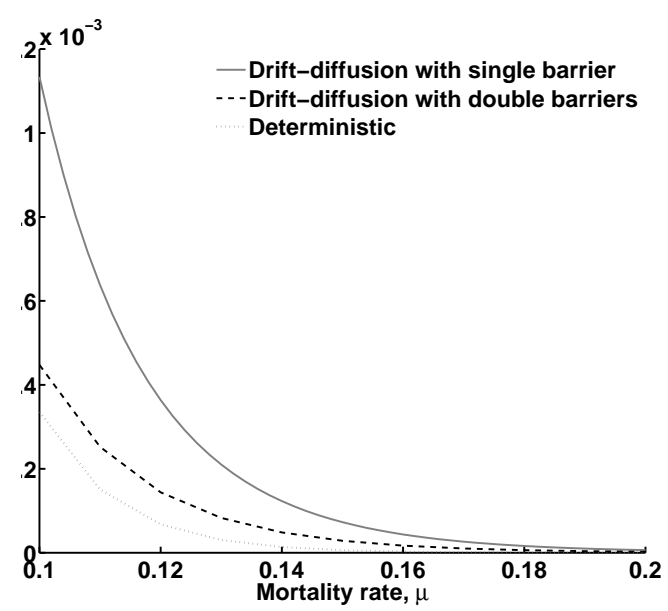

d)

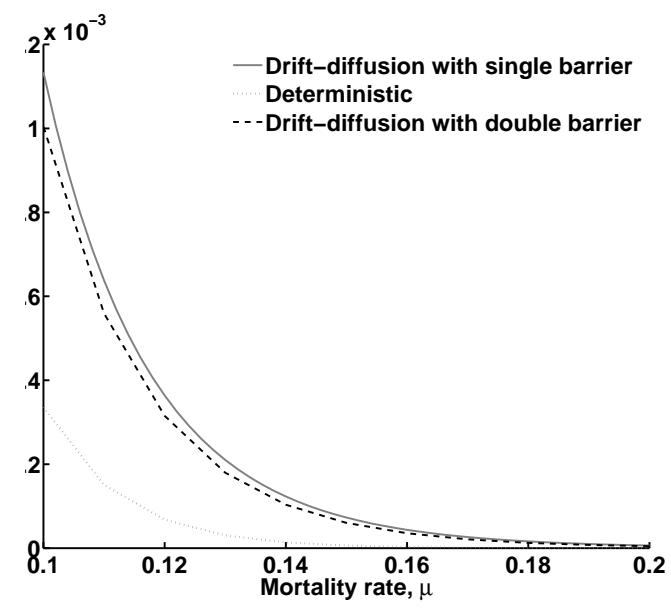

Figure 3: Examples of the effect of an additional absorbing barrier on the probability of recruitment, for the drift-diffusion model of [23]. In all graphs $\sigma=5$ and a) $M_{0}=2, r=5$, b) $M_{0}=2, r=2.5$, c) $M_{0}=10, r=5$, d) $M_{0}=10, r=2.5$. 
Taking the same simple mortality model as [23], we can arrive at the probability of maturation,

$$
\begin{aligned}
P_{\text {mat }}(r, \sigma) & =\int_{0}^{\infty} f_{\hat{t}_{\text {mat }}}(t) \exp (-\mu t) d t \\
& =\int_{0}^{\infty} \exp \left\{\frac{M_{\text {mat }} r}{\sigma^{2}}-\frac{1}{2}\left(\frac{r}{\sigma}\right)^{2} t-\mu t\right\} \sum_{n=-\infty}^{\infty} g\left(t ; b_{n}\right) d t
\end{aligned}
$$

We can now repeat the results of [23], to assess whether the inclusion of an absorbing barrier at "zero" alters the conclusions. Figure 3 shows the results for the double barrier hitting time problem, for an initial mass of $M_{0}=2$ (a) and b) and $M_{0}=10$ (c) and d) in comparison to the single barrier problem.

For an initial larval mass of $M_{0}=2$ the addition of the second absorbing barrier can change the results of [23], especially in the $r=2.5$ case: stochasticity is not necessarily beneficial to recruitment because it increases the probability of absorption at the lower barrier. For $M_{0}=10$, the additional barrier does not significantly affect the results for $r=5$, and has only a small effect on the results for $r=2.5$.

a)

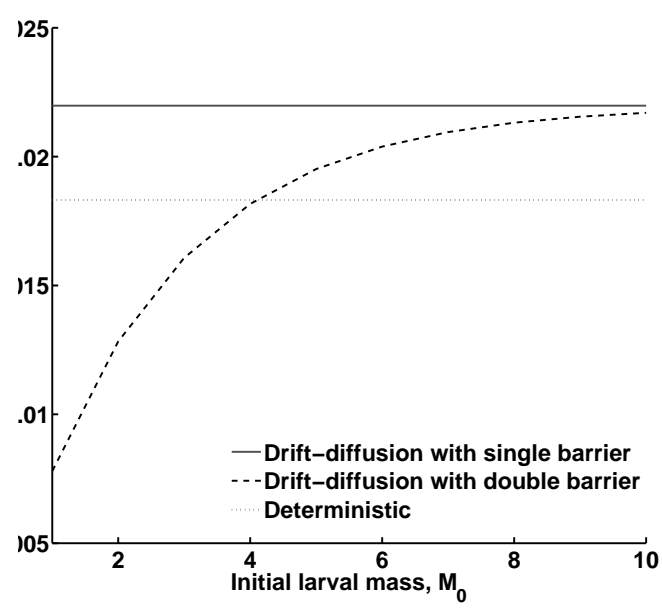

b)

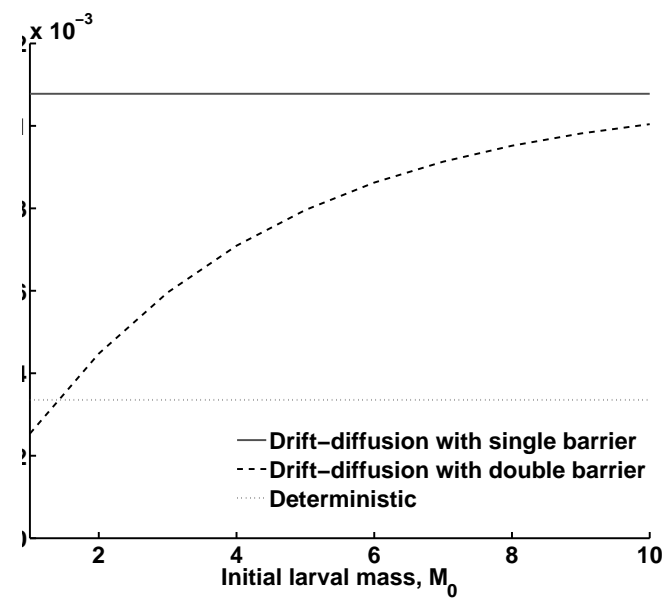

Figure 4: The dependence of the probability of recruitment on initial larval mass $M_{0}$ for the double barrier hitting time problem. In both graphs $\mu=0.1, \sigma=5$ and a) $r=5$, b) $r=2.5$.

The effect of the lower absorbing barrier is highly dependent on the value of $M_{0}$, even within a small range (as shown in Figure 4). The parameters used in this paper are chosen to be broadly representative of a fish larva reaching recruitment mass after an average of 40 days [23]. Values for $M_{0}$ (relative to a fixed $M_{\text {mat }}=200$ ) in the literature can range over at least two orders of magnitude e.g. $M_{0}=0.12$ for bay anchovy Anchoa mitchilli larvae reaching metamorphosis in 32 days, $M_{0}=22.3$ for European plaice Pleuronectes platessa larvae reaching metamorphosis in 100 days [11] (www.fishbase.org). For species and ecological scenarios where the starvation of larvae 
is known to be an ecologically relevant process, the possibility of absorption at the lower barrier can be included using the above methods. However, our overall conclusions regarding the role of superdiffusive growth in the recruitment process are qualitatively unaffected.

\section{References}

[1] D. Applebaum. Lévy Processes and Stochastic Calculus. CUP, Cambridge, 2004.

[2] F. Bartumeus. Lévy processes in animal movement: an evolutionary hypothesis. Fractals $15(2)(2007), 151-162$.

[3] F. Bartumeus, J. Catalan, U. L. Fulco, M. L. Lyra, G. M. Viswanathan.Optimizing the encounter rate in biological interactions: Lévy versus Brownian strategies. Phys. Rev. Let. 88(9) (2002), 097901.

[4] F. Bartumeus, M. G. E. da Luz, G. M. Viswanathan, J. Catalan. Animal search strategies: a quantitative random walk analysis. Ecology 86 (2005), 3078-3087.

[5] S. Benhamou. How many animals really do the Lévy walk? Ecology 88(8) (2007), $1962-$ 1969.

[6] R. C. Chambers, E. A. Trippel. Early Life History and Recruitment in Fish Populations. Chapman and Hall, London, 1997.

[7] S. Condamin, P. Bénichou, V. Tejedor, R. Voituriez, J. Klafter. First-passage times in complex scale-invariant media. Nature 450 (2007), 77-80.

[8] J. D. Currey, J. W. Pitchford, P. D. Baxter. Variability of the mechanical properties of bone, and its evolutionary consequences. J. R. Soc. Interface 4(12) (2007), 127-135.

[9] D. H. Cushing, J. W. Horwood. The growth and death of fish larvae. J. Plankton Res. 16(3) (1994), 291-300.

[10] A. M. Edwards, R. A. Phillips, N. W. Watkins, M. P. Freeman, E. J. Murphy, V. Afanasyev, S. V. Buldyrev, M. G. E da Luz, E. P. Raposo, H. E. Stanley, G. M. Viswanathan. Revisiting Lévy flight search patterns of wndering albatrosses, bumblebees and deer. Nature 449 (2007), 1044-1047.

[11] R. Froese, D. Pauly. Fishbase 2000: concepts, design and data sources. ICLARM, Los Baos, Laguna, Philippines, 344 (2000), 344 p.

[12] P. S. Galbraith, H. I. Browman, R. G. Racca, A. B. Skiftesvik, J. F. Saint-Pierre. Effect of turbulence on the energetics of foraging in Atlantic cod Gadus morhua larvae. Mar. Ecol. Prog. Ser. 281 (2004), 241-257. 
[13] A. James, P. D. Baxter, J. W. Pitchford. Modelling predation as a capped rate stochastic process, with applications to fish recruitment. J. R. Soc. Interface 2 (2005), 477-487.

[14] N. L. Johnson, S. Kotz, N. Balakrishnan. Continuous univariate distributions. Vol 1. John Wiley \& Sons, Inc., New York, 1994.

[15] X. S. Lin. Double barrier hitting time distributions with applications to exotic options. Insurance: Mathematics and Economics 23 (1998), 45-58.

[16] R. G. Lough, E. A. Broughton. Development of micro-scale frequency distributions of plankton for inclusion in foraging models of larval fish. Results from a Video Plankton Recorder. J. Plankton Res. 29(1) (2007), 7-17.

[17] Q. Lv, J. W. Pitchford. Stochastic von Bertalanffy models, with applications to fish recruitment. J. Theor. Biol. 244 (2007), 640-655.

[18] B. R. Mackenzie, T. Kiorboe. Encounter rates and swimming behavior of pause-travel and cruise larval fish predators in calm and turbulent laboratory environments. Limnology and Oceanography 40(7) (1995), 1278-1289.

[19] A. G. Pakes. A hitting time for Lévy processes, with application to dams and branching processes. Annales de le Faculte des Sciences de Toulouse 5(3) (1996), 521-544.

[20] P. Pepin, J. F. Dower, F. Davidson. Spatially explicit study of prey-predator interactions in larval fish: assessing the influence of food and predator abundance on growth and survival. Fish. Oceanogr. 12 (2003), 19-33.

[21] J. W. Pitchford, J. Brindley. Prey patchiness, predator survival and fish recruitment. Bull. Math. Biol. 63 (2001), 527-546.

[22] J. W. Pitchford, A. James, J. Brindley. Optimal foraging in patchy turbulent environments. Mar. Ecol. Prog. Ser. 256 (2003), 99-110.

[23] J. W. Pitchford, A. James, J. Brindley. Quantifying the effects of individual and environmental variability in fish recruitment. Fish. Oceanogr. 14(2) (2005), 156-160.

[24] M. J. Plank, A. James. Optimal foraging: Lévy pattern or process? J. R. Soc. Interface 5 (2008), 1077-1086.

[25] J. J. Ruzicka, S. M. Gallagher. The salutatory search behavior of larval cod (Gadus morhua). Deep-sea Research part II- Topical Studies in Oceanography 53(23-24) (2006), 2735-2757.

[26] D. W. Sims, D. Righton, J. W. Pitchford. Minimizing errors in identifying Lévy flight behaviour of organisms. J. Animal Ecol. 76(2) (2007), 222-229. 
[27] D. W. Sims, E. J. Southall, N. E. Humphries, G. C. Hays, C. J. A. Bradshaw, J. W. Pitchford, A. James, M. Z. Ahmed, A. S. Brierly, M. A. Hindell, D. Morritt, M. K. Musyl, D. Righton, E. L. C. Shepard, V. J. Wearmouth, R. P. Wilson, M. J. Witt, J. D. Metcalfe. Scaling laws of marine predator search behaviour. Nature 451 (2008), 1098-U5.

[28] G. M. Viswanathan, S. V. Buldyrev, S. Havlin, M. G. E. da Luz, E. P. Raposo, H. E. Stanley. Optimizing the success of random searches. Nature 401 (1999), 911-914.

[29] G. M. Viswanathan, V. Afanasyev, S. V. Buldyrev, S. Havlin, M. G. E. da Luz, E. P. Raposo, H. E. Stanley. Lévy flights in random searches. Physica A 282 (2000), 1-12. 